\title{
Hamilton Equations, Commutator, and Energy Conservation ${ }^{\dagger}$
}

\author{
Weng Cho Chew ${ }^{1, *(\mathbb{C}}$, Aiyin Y. Liu ${ }^{2}\left(\mathbb{D}\right.$, Carlos Salazar-Lazaro ${ }^{3}\left(\mathbb{D}\right.$, Dong-Yeop Na ${ }^{1}(\mathbb{D}$ \\ and Wei E. I. Sha ${ }^{4}(\mathbb{D}$ \\ 1 College of Engineering, Purdue University, West Lafayette, IN 47907, USA; na32@purdue.edu \\ 2 College of Engineering, University of Illinois at Urbana-Champaign, Urbana, IL 61820, USA; \\ ayliu.uiuc@gmail.com \\ 3 Physics Department, University of Illinois at Urbana-Champaign, Urbana, IL 61820, USA; \\ slzrlzr2@illinois.edu \\ 4 College of Information Science and Electronic Engineering, Zhejiang University, Hangzhou 310058, China; \\ weisha@zju.edu.cn \\ * Correspondence: wcchew@purdue.edu \\ + Based on the talk presented at the 40th Progress In Electromagnetics Research Symposium (PIERS, Toyama, \\ Japan, 1-4 August 2018).
}

Received: 12 September 2019; Accepted: 3 December 2019; Published: 9 December 2019

\begin{abstract}
We show that the classical Hamilton equations of motion can be derived from the energy conservation condition. A similar argument is shown to carry to the quantum formulation of Hamiltonian dynamics. Hence, showing a striking similarity between the quantum formulation and the classical formulation. Furthermore, it is shown that the fundamental commutator can be derived from the Heisenberg equations of motion and the quantum Hamilton equations of motion. Also, that the Heisenberg equations of motion can be derived from the Schrödinger equation for the quantum state, which is the fundamental postulate. These results are shown to have important bearing for deriving the quantum Maxwell's equations.
\end{abstract}

Keywords: quantum mechanics; commutator relations; Heisenberg picture

\section{Introduction}

In quantum theory, a classical observable, which is modeled by a real scalar variable, is replaced by a quantum operator, which is analogous to an infinite-dimensional matrix operator. A quantum operator that represents an observable endows the observable with probabilistic properties such as a mean and a variance (or fluctuation from its mean as it is measured by the standard deviation from its mean).

In order to associate an operator with a mean, a quantum system is described by a quantum state or a state vector $|\Psi\rangle$. For instance, given an operator $\hat{p}$ and a quantum state described by the state vector $|\Psi\rangle$, the mean of the operator is its expectation value given by $\bar{p}=\langle\Psi|\hat{p}| \Psi\rangle$ in Dirac's notation. This is entirely analogous to the linear algebra notation $\bar{p}=\Psi^{\dagger} \cdot \overline{\mathbf{p}} \cdot \mathbf{\Psi}$. This associates a real scalar number $\bar{p}$ with a Hermitian operator $\hat{p}$ in the given quantum state $\Psi$. The fluctuation from its mean or its standard deviation is given by $\sigma_{p}=\left(\left\langle\Psi\left|\hat{p}^{2}\right| \Psi\right\rangle-\bar{p}^{2}\right)^{1 / 2}$.

For example, classically, a particle is described with a position variable $q$ and a momentum variable $p$. In quantum theory, the position variable is replaced by a position operator $\hat{q}$, and the momentum variable is replaced by a momentum operator $\hat{p}$. In the quantum world, the position operator $\hat{q}$ and the momentum operator $\hat{p}$ do not commute or $\hat{p} \hat{q} \neq \hat{q} \hat{p}$. The fundamental postulate of quantum mechanics is the canonical commutator that [1-4]:

$$
[\hat{q}, \hat{p}]=\hat{q} \hat{p}-\hat{p} \hat{q}=i \hbar \hat{I}
$$


where the square bracket represents the definition of a commutator. Sometimes, this is called Dirac's fundamental postulate [1]. Many properties of quantum physics, such as the uncertainty principle, can be derived from this postulate. Moreover, one can derive the representation of the momentum operator $\hat{p}$ in terms of the coordinate operator $\hat{q}$. Hence, the fundamental postulate is important for quantum theory as well as the quantization of electromagnetic fields where the quantum fluctuation of electromagnetic fields is important.

In this note, we shall show that the fundamental commutator can be derived from the Heisenberg equations of motion, and the quantum Hamilton equations. Heisenberg equations can be derived from the quantum state equation, and quantum Hamilton equations can be derived from energy conservation argument. The fundamental postulate of quantum theory is the quantum state equation, which was first proposed by Schrödinger, based on the energy conservation argument.

Furthermore, it can be shown that quantum Hamilton equations can be derived from the Heisenberg equations of motion after invoking the fundamental commutator [3]. The quantum Hamilton equations are very similar in form to the classical Hamilton equations. As such, there is usually a clear analogy and a mathematical homomorphism between the classical and quantum theories of many systems, such as electromagnetic fields. This streamlines the derivation of many quantum equations of motion. It also explains why the equations of motion for quantum electromagnetics resemble those of classical electromagnetics [5].

Using this mathematical homomorphism, one can derive the quantum Maxwell equations without using mode decomposition (or Fourier decomposition), a procedure followed in most textbooks [6-8]. Maxwell's equations can be quantized directly in the coordinate space. Moreover, it is easy to add impressed sources to Maxwell's equations and quantize them, as has been shown in [5].

For a set of discrete oscillators coupled to each other, the canonical commutator of (1) (see [6]) is:

$$
\left[\hat{q}_{i}, \hat{p}_{j}\right]=i \hbar \hat{I} \delta_{i j}
$$

In the continuum limit, when the variables $\hat{q}_{i}$ and $\hat{p}_{i}$ become fields $\hat{\Phi}\left(\mathbf{r}^{\prime}, t\right)$ and $\hat{\Pi}_{s}(\mathbf{r}, t)$, respectively, then the commutator becomes [5]:

$$
\left[\hat{\Phi}\left(\mathbf{r}^{\prime}, t\right), \hat{\Pi}_{s}(\mathbf{r}, t)\right]=i \hbar \delta\left(\mathbf{r}-\mathbf{r}^{\prime}\right) \hat{I}
$$

or the Kronecker delta function becomes a Dirac delta function in the continuum limit. It can be shown that these commutators induce derivatives with respect to operators and give rise to the quantum Hamilton equations. Using the above, the quantum Maxwell equations can be derived [5].

\section{Classical Hamiltonian of an Oscillator}

A general classical oscillator, including the anharmonic oscillator, has a Hamiltonian given by:

$$
H=\frac{1}{2 m} p^{2}+V(q)
$$

where $p$ and $q$ are functions of time $t$. Its multi-variable differential can be written as:

$$
\delta H=\frac{\partial H}{\partial p} \delta p+\frac{\partial H}{\partial q} \delta q .
$$

The above implies that:

$$
\frac{d H}{d t}=\frac{\partial H}{\partial p} \frac{d p}{d t}+\frac{\partial H}{\partial q} \frac{d q}{d t} .
$$

In order for the above to be zero for energy conservation, then:

$$
\frac{d p}{d t}=-C \frac{\partial H}{\partial q}, \quad \frac{d q}{d t}=C \frac{\partial H}{\partial p} .
$$


The above are the infinitely many possible energy conserving equations of motion for this problem. They are similar to the Hamilton equations determined up to a factor $C$. The reason is that the clock is not defined in a kinematic system, and all of these systems moving with different clocks will satisfy energy conservation. However, we can fix the clock by invoking the definition of velocity in terms of momentum, namely that:

$$
\frac{d q}{d t}=\frac{p}{m}
$$

i.e., the connection between velocity and momentum, which can be derived from the first equation of (7) with (4). For this, we need to pick $C=1$.

The above can be generalized to the case when $C=C(t)$, viz., it is time dependent. This is physically equivalent to a time varying clock. Again, we can use (8) to calibrate our clock. When $C=C(t)$, (7) is mathematically equivalent to the case when the general energy conserving solution is such that:

$$
\frac{\partial H}{\partial p} \frac{d p}{d t}=f(t), \quad \text { and } \quad \frac{\partial H}{\partial q} \frac{d q}{d t}=-f(t)
$$

which is another way to express an energy conserving solution.

Furthermore, (7) can be rewritten as:

$$
\frac{1}{C(t)} \frac{d p}{d t}=-\frac{\partial H}{\partial q}, \quad \frac{1}{C(t)} \frac{d q}{d t}=C \frac{\partial H}{\partial p} .
$$

Then, the time stretching technique, similar to the coordinate stretching technique [9-13], can be used to rewrite the above as:

$$
\frac{d p}{d t^{\prime}}=-\frac{\partial H}{\partial q}, \quad \frac{d q}{d t^{\prime}}=\frac{\partial H}{\partial p} .
$$

where $t^{\prime}=\int_{0}^{t} C\left(t^{\prime \prime}\right) d t^{\prime \prime}$. To agree with laboratory experiments, the time stretching variable $C(t)=1$.

\section{Quantum Hamiltonian}

In the quantum world, $p$ and $q$, the canonical conjugate variables, are elevated to be quantum operators. Hence, (4) becomes:

$$
\hat{H}=\frac{1}{2 m} \hat{p}^{2}+\hat{V}(\hat{q}) .
$$

Since functions of an operator argument are operators themselves, $\hat{V}$ and $\hat{H}$ are now quantum operators. This Hamiltonian operator acts on a state vector $|\psi\rangle$ in the manner of the Schrödinger equation, viz.,

$$
\hat{H}|\psi\rangle=i \hbar \partial_{t}|\psi\rangle
$$

where $|\psi\rangle$ is a vector that indicates the state of the quantum system with the normalization condition, i.e., $\langle\psi \mid \psi\rangle=1$. Equation (13) is a fundamental postulate of quantum theory. It cannot be derived, but it is based on the postulate of Schrödinger (Later, this equation for the quantum state was used for quantum Hamiltonians for different quantum systems, e.g., the Dirac equation for relativistic electrons. It has also been used for the quantum Maxwell equations [5]. In order not to confuse this with the Schrödinger equation for wave functions in an atom, for instance, we will call this Schrödinger's quantum state equation.). In addition, for energy conservation, $\hat{H}$, which represents the total energy of the system, is time independent, and $|\psi\rangle$ solves: (13) is of the form

$$
|\psi(t)\rangle=e^{-i \frac{\hat{H}}{\hbar} t}\left|\psi_{0}\right\rangle
$$


where $|\psi(t=0)\rangle=\left|\psi_{0}\right\rangle$.

The requirement of a quantum system is that the expectation value of the quantum operators, which are the quantum representation of an observable or canonical dynamic variable, be equal to their classical analog. For instance, the expectation value of the Hamiltonian operator $\hat{H}$ is:

$$
H=\langle\psi(t)|\hat{H}| \psi(t)\rangle .
$$

With (14) substituted into (15), it indeed can be shown that $H$ is independent of time. In other words,

$$
H=\left\langle\psi_{0}\left|e^{i \frac{\hat{H}}{\hbar} t} \hat{H} e^{-i \frac{\hat{H}}{\hbar} t}\right| \psi_{0}\right\rangle=\text { constant }
$$

In the above, $\hat{H}$ commutes with a function of $\hat{H}$, namely $e^{-i \frac{\hat{H}}{\hbar} t}$, and hence, the above time dependence cancels, yielding a constant of time implying energy conservation. So far, nothing has been said of the commutators in quantum theory. Therefore, the assumption that $\hat{H}$ is time independent is consistent with energy conservation.

One can show further that given an operator $\hat{o}_{S}$ in the Schrödinger picture, it has the expectation value given by:

$$
\begin{aligned}
\bar{o}(t)=\left\langle\psi(t)\left|\hat{o}_{s}\right| \psi(t)\right\rangle & =\left\langle\psi_{0}\left|e^{i \frac{\hat{H}}{\hbar}} t \hat{o}_{s} e^{-i \frac{\hat{H}}{\hbar} t}\right| \psi_{0}\right\rangle \\
& =\left\langle\psi_{0}\left|\hat{o}_{h}(t)\right| \psi_{0}\right\rangle
\end{aligned}
$$

where the operator in the Heisenberg picture emerges [4],

$$
\hat{o}_{h}(t)=e^{i \frac{\hat{H}}{\hbar}} \hat{o}_{s} e^{-i \frac{\hat{H}}{\hbar} t}
$$

and $\hat{o}_{S}$ in the Schrödinger picture is time independent. In the following, we will eliminate the subscript $h$. When an operator is a function of time, it is understood to be in the Heisenberg picture. Moreover, it can be shown that the equation of motion for $\hat{o}$ in the Heisenberg picture takes the form of:

$$
\frac{\mathrm{d} \hat{o}(t)}{\mathrm{d} t}=-\frac{i}{\hbar}[\hat{o}(t), \hat{H}]
$$

where $[\hat{A}, \hat{B}]=\hat{A} \hat{B}-\hat{B} \hat{A}$ is the commutator. The above is the Heisenberg equation of motion.

Given the fundamental commutator relation, $[\hat{q}, \hat{p}]=i \hbar \hat{I}$, it can be shown that $[3-5]$ :

$$
\begin{aligned}
{[\hat{q}, \hat{p}]=i \hbar \hat{I} } & \Longrightarrow\left[\hat{p}, \hat{q}^{n}\right]=-i n \hat{q}^{n-1} \hbar=-i \hbar\left(\frac{\partial \hat{q}^{n}}{\partial \hat{q}}\right), \\
& \Longrightarrow\left[\hat{q}, \hat{p}^{n}\right]=i n \hat{p}^{n-1} \hbar=i \hbar\left(\frac{\partial \hat{p}^{n}}{\partial \hat{p}}\right) .
\end{aligned}
$$

The above introduces the concept of a derivative with respect to to an operator, namely that $\frac{\partial \hat{o}^{n}}{\partial \hat{o}}=n \hat{o}^{n-1}$. We will digress to discuss this in more detail.

It is to be noted that the above is derived by induction, or recursion: Given that:

$$
\left[\hat{p}, \hat{q}^{n-1}\right]=-i n \hat{q}^{n-2} \hbar
$$

and the commutator $[\hat{q}, \hat{p}]=i \hbar \hat{I}$, it can be shown that:

$$
\left[\hat{p}, \hat{q}^{n}\right]=-i n \hat{q}^{n-1} \hbar .
$$

The reverse of the above procedure is also true. 


\subsection{A Word on the Derivative with Respect to the Operator}

In the above, the derivative of an operator with respect to to an operator has meaning only when the operator is acting on its eigenstate (eigenvector). Then, the operator can be treated as a scalar number, which is the eigenvalue associated with the particular eigenvector. Assume that a quantum state is expanded in terms of a set of eigenvectors, which is given by:

$$
|\psi\rangle=\sum_{i} d_{i}\left|q_{i}\right\rangle
$$

where $\left|q_{i}\right\rangle$ are the eigenvectors of $\hat{q}$, then it follows that:

$$
\begin{aligned}
\partial_{\hat{q}} \hat{q}^{n}|\psi\rangle=\frac{\partial \hat{q}^{n}}{\partial \hat{q}}|\psi\rangle=\sum_{i} \frac{\partial q_{i}^{n}}{\partial q_{i}} d_{i}\left|q_{i}\right\rangle & =\sum_{i} n q_{i}^{n-1} d_{i}\left|q_{i}\right\rangle \\
& =n \hat{q}^{n-1}|\psi\rangle
\end{aligned}
$$

where $\partial_{\hat{q}}$ is an alternative way of denoting the derivative with respect to an operator, and here, $\partial_{\hat{q}} \hat{q}^{n}=n \hat{q}^{n-1}$. The above implies that in evaluating $\partial_{\hat{q}} \hat{q}^{n}$, we will let this operator $\hat{q}^{n}$ act on the state vector expanded in terms of $\left|q_{i}\right\rangle$, the eigenvectors of $\hat{q}$, and then take the derivative with respect to the eigenvalue $q_{i}$ in the preceding equation. Hence, $\partial_{\hat{q}} \hat{q}^{n}$ only produces another operator $n \hat{q}^{n-1}$.

The above is in agreement with a perturbation expansion or the first variation of $\hat{q}$, so that:

$$
(\hat{q}+\delta \hat{q})^{n}
$$

It expands to only first order to:

$$
\hat{q}^{n}+n \hat{q}^{n-1} \delta \hat{q}
$$

only if

$$
[\hat{q}, \delta \hat{q}]=0
$$

or they commute and share the same set of eigenvectors. In other words, the same set of eigenvectors diagonalizes $\hat{q}$ and $\delta \hat{q}$ simultaneously. That $\delta \hat{q}$ and $\hat{q}$ share the same set of eigenvectors is in fact implied in the derivation of (25). The commutator in (28) is important in allowing a simple definition of the derivative with respect to an operator.

\section{Quantum Hamilton Equations from Heisenberg Equations}

In general, if $P(p)$ and $Q(q)$ are polynomial functions, then from: (20) and (21)

$$
\begin{aligned}
& {[\hat{p}, \hat{Q}(\hat{q})]=-i \hbar \frac{\partial \hat{Q}(\hat{q})}{\partial \hat{q}}} \\
& {[\hat{q}, \hat{P}(\hat{p})]=i \hbar \frac{\partial \hat{P}(\hat{p})}{\partial \hat{p}} .}
\end{aligned}
$$

To get the quantum Hamilton equations, we consider a particular class of Hamiltonians as follows.

\section{Sum Separable Hamiltonians}

In this case,

$$
H(p, q)=P(p)+Q(q)
$$

where $P$ and $Q$ are polynomial functions, or that:

$$
P(p)=\sum_{n} a_{n} p^{n}, \quad Q(q)=\sum_{m} b_{m} q^{m} .
$$


The above can be elevated to $\hat{P}$ and $\hat{Q}$ after elevating the canonical variables $p$ and $q$ to quantum operators. Then, a generic form of the quantum Hamiltonian, in index or Einstein notation, is:

$$
\hat{H}=a_{n} \hat{p}^{n}+b_{m} \hat{q}^{m} .
$$

Then, it is quite easy to show that the Heisenberg equations imply the quantum Hamilton equations, namely that:

$$
\begin{aligned}
& \frac{d \hat{p}}{d t}=-\frac{i}{\hbar}[\hat{p}, \hat{H}]=-\frac{\partial \hat{H}}{\partial \hat{q}}, \\
& \frac{d \hat{q}}{d t}=-\frac{i}{\hbar}[\hat{q}, \hat{H}]=\frac{\partial \hat{H}}{\partial \hat{p}} .
\end{aligned}
$$

In other words, the fundamental commutator and Heisenberg equations imply the quantum Hamilton equations as,

$$
\frac{\mathrm{d} \hat{q}}{\mathrm{~d} t}=\frac{\partial \hat{H}(\hat{p}, \hat{q})}{\partial \hat{p}}, \quad \frac{\mathrm{d} \hat{p}}{\mathrm{~d} t}=-\frac{\partial \hat{H}(\hat{p}, \hat{q})}{\partial \hat{q}} .
$$

It is to be noted that since the derivations in (20) and (21) can be reversed, then one can start with the quantum Hamilton equations and derive the Heisenberg equations of motion in (34) and (35).

\section{Quantum Hamilton Equations and Energy Conservation}

In the above, we have shown that quantum Hamilton equations can be derived from the Heisenberg equations with the fundamental commutator as given in (1). It will be curious to see if the quantum Hamilton equations can be derived from energy conservation argument alone. Again, we look at sum separable Hamiltonians.

\subsection{Sum Separable Hamiltonian}

For sum separable Hamiltonians, one assumes that a Hamiltonian is of the form:

$$
\hat{H}(\hat{p}, \hat{q})=\hat{P}(\hat{p})+\hat{Q}(\hat{q}) .
$$

Then, a generic form of the Hamiltonian in index notation is:

$$
\hat{H}=a_{n} \hat{p}^{n}+b_{m} \hat{q}^{m} .
$$

By taking the first variation, assuming the following commutators:

$$
\begin{array}{r}
{[\hat{p}, \delta \hat{p}]=0,} \\
{[\hat{q}, \delta \hat{q}]=0,}
\end{array}
$$

it is quite easy to show that:

$$
\delta \hat{H}=\frac{\partial \hat{H}}{\partial \hat{p}} \delta \hat{p}+\frac{\partial \hat{H}}{\partial \hat{q}} \delta \hat{q}
$$

or that:

$$
\frac{d \hat{H}}{d t}=\frac{\partial \hat{H}}{\partial \hat{p}} \frac{d \hat{p}}{d t}+\frac{\partial \hat{H}}{\partial \hat{q}} \frac{d \hat{q}}{d t} .
$$

In the above, there are derivatives with respect to the quantum operators $\hat{p}$ and $\hat{q}$. These derivatives are defined as in Section 3.1. Hence, Equation (36), the quantum Hamilton equations, can be substituted into the above equation. Then, it is easily shown that $\frac{d \hat{H}}{d t}=0$, implying energy conservation. 
Since the above Hamilton Equations (36) have been derived from the Heisenberg equations and fundamental commutator and they are consistent with energy conservation, or in short,

$$
\begin{array}{r}
{[\hat{q}, \hat{p}]=i \hbar \hat{I} \text { plus Heisenberg equations }} \\
\Longrightarrow \text { energy conservation }
\end{array}
$$

\subsection{Quantum Hamilton Equations from Energy Conservation}

It is interesting to ask the converse: Does energy conservation imply the quantum Hamilton equations? Before doing so, we shall elaborate more on (40) and (41). We can also write down the expression for (40) more explicitly using (37), namely:

$$
\delta \hat{H}=n a_{n} \hat{p}^{n-1} \delta \hat{p}+m b_{m} \hat{q}^{m-1} \delta \hat{q} .
$$

It is to be noted that the commutators (39) do not restrict the variations of $\delta \hat{p}$ and $\delta \hat{q}$ in (43). To see this, one can take the second term on the right-hand side of the above and let it operate on (24). Then, the new state vector:

$$
|\Phi\rangle=m b_{m} \hat{q}^{m-1} \delta \hat{q}|\Psi\rangle=m b_{m} \sum_{i} q_{i}^{m-1} \delta q_{i} d_{i}\left|q_{i}\right\rangle
$$

Because $\delta q_{i}$ is arbitrary and $\left|q_{i}\right\rangle$ is complete, this new state vector $|\Phi\rangle$ can be made to point in any "arbitrary" direction in Hilbert space. A similar thought experiment can be done on the first term of (43). In other words, the commutators (39) do not diminish the degree of freedom in the variation of (43). Moreover, due to the commutators (39), the order of $\delta \hat{p}$ and $\delta \hat{q}$ is unimportant in the above, and the commutators give rise to the very simple equation given by (41). Therefore, using (41), a set of simple possible solutions for an energy conserving system is:

$$
\text { Energy Conservation } \Longrightarrow \frac{d \hat{p}}{d t}=-C \frac{\partial \hat{H}}{\partial \hat{q}}, \quad \frac{d \hat{q}}{d t}=C \frac{\partial \hat{H}}{\partial \hat{p}} \text {. }
$$

They are similar to the Hamilton equations within a multiplicative constant $C$. There are infinitely many possibilities, because the clock is not calibrated. However, just as the classical case as shown in (7), we can apply the above to a simple oscillator and use a simple case that $\frac{d \hat{q}}{d t}=\frac{\hat{p}}{m}$ to calibrate our clock to give $C=1$. As a result, the quantum Hamilton equations can also be derived from energy conservation.

The above, as before, can be generalized to the case when $C=C(t)$, which is more general than the above. Again, the same physical interpretation applies in this case, as in the classical case. We have a time varying clock that can be calibrated to a time invariant clock, meanwhile, in agreement with the clock in the real world.

\section{Fundamental Commutator from Energy Conservation}

The Heisenberg equations of motion are also energy conserving as they are derived from the quantum state Equation (13). Hence, both the Hamilton equations and Heisenberg equations of motion can be derived without invoking the fundamental commutator, but merely from energy conservation alone. Next, we shall show that the fundamental commutator can actually be derived from the quantum Hamilton equations and Heisenberg equations of motion.

We can start with one of the quantum Hamilton equations that follows from energy conservation argument after calibrating the clock. This is:

$$
\frac{d \hat{q}}{d t}=\partial_{\hat{p}} \hat{H} .
$$


Next, we look at the Heisenberg equations of motion, one of which is:

$$
\frac{d \hat{q}}{d t}=A[\hat{q}, \hat{H}]
$$

where we define $A=-i / \hbar$. From (46) and (47), it follows that

$$
\partial_{\hat{p}} \hat{H}=A[\hat{q}, \hat{H}] .
$$

By writing the Hamiltonian as a polynomial, then from the above, taking the $m$-th term of the polynomial, it is quite easy to show that

$$
\partial_{\hat{p}} \hat{p}^{m}=m \hat{p}^{m-1}=A\left[\hat{q}, \hat{p}^{m}\right], \quad m=1, \ldots
$$

By setting $m=1$ in the above, then

$$
1=A[\hat{q}, \hat{p}] \rightarrow[q, p]=i \hbar
$$

which is the fundamental commutator. Therefore preceding argument shows that the fundamental commutator can be derived from, or is consistent with, quantum Hamilton equations and Heisenberg equations of motion, which are energy conserving equations.

One of the fundamental postulates of quantum theory is (13), which cannot be derived. It is the equation of motion for the quantum state. It was first proposed by Schrodinger, and later used to describe all quantum systems, include Dirac's equation for relativistic electrons.

The fundamental commutator is important for energy conservation, and it can be generalized to the quantization of electromagnetic fields [5]. However, it is intimately related to energy conservation.

\section{Conclusions}

1. The fundamental commutator can be derived from Heisenberg equations of motion and the quantum Hamilton equations.

2. The quantum Hamilton equations can be derived by energy conservation argument after the calibration of the clock.

3. The quantum Hamilton equations can also be derived from Heisenberg equations of motion, together with the fundamental commutator. Also, the quantum Hamilton equations thus derived are shown to be energy conserving.

4. The important fundamental postulate of quantum theory is that first put forth by Schrödinger, for the quantum state Equation (13). One can derive the Heisenberg equations of motion from the quantum state equation.

Author Contributions: Conceptualization, W.C.C.; formal analysis, W.C.C., A.Y.L., C.S.-L., D.-Y.N., and W.E.I.S.; funding acquisition, W.C.C.; investigation W.C.C.; methodology, W.C.C.; project administration, W.C.C.; supervision, W.C.C.

Funding: This research was funded by the National Science Foundation 1818910 award and a start up fund at Purdue University. W.C.C. was supported by DVSSHong Kong University during the summer of 2019.

Acknowledgments: W.C.C. would like to thank numerous discussions with Fuchun Zhang, Guanhua Chen, and Jian Wang of Hong Kong Uand Hong Guo of McGill U when we were working on the Area of Excellence project on Theory, Modeling, Simulation of Emerging Electronics funded by CERGof the Hong Kong government.

Conflicts of Interest: The authors declare no conflict of interest.

\section{Abbreviations}

The following abbreviations are used in this manuscript: 


\section{References}

1. Dirac, P.A.M. The Principles of Quantum Mechanics; Number 27 in The International Series of Monographs on Physics; Oxford University Press: Oxford, UK, 1981.

2. Sakurai, J.J. Advanced Quantum Mechanics; Pearson Education India: Bengaluru, India, 1967.

3. Louisell, W.H.; Louisell, W.H. Quantum Statistical Properties of Radiation; Wiley: New York, NY, USA, 1973; Volume 7.

4. Gottfreid, K.; Yan, T.M. Quantum Mechanics: Fundamentals; Springer: Berlin, Germany, 2003.

5. Chew, W.C.; Liu, A.Y.; Salazar-Lazaro, C.; Sha, W.E.I. Quantum electromagnetics: A new look-Part I and Part II. IEEE J. Multiscale Multiphys. Comput. Tech. 2016, 1, 73-97. [CrossRef]

6. Haken, H. Quantum Field Theory of Solids, an Introduction; North-Holland Publishing Company: Amsterdam, The Netherlands, 1976.

7. Mandel, L.; Wolf, E. Optical Coherence and Quantum Optics; Cambridge University Press: Cambridge, UK, 1995.

8. Cohen-Tannoudji, C.; Dupont-Roc, J.; Grynberg, G. Interactions, Atom-Photon: Basic Processes and Applications; Willey Interscience: Hoboken, NJ, USA, 1992.

9. Chew, W.C.; Weedon, W.H. A 3D perfectly matched medium from modified Maxwell's equations with stretched coordinates. Microw. Opt. Technol. Lett. 1994, 7, 599-604. [CrossRef]

10. Chew, W.C.; Jin, J.; Michielssen, E. Complex coordinate stretching as a generalized absorbing boundary condition. Microw. Opt. Technol. Lett. 1997, 15, 363-369. [CrossRef]

11. Harrington, R.F. Time-Harmonic Electromagnetic Fields; McGraw-Hill: New York, NY, USA, 1961.

12. Balanis, C.A. Advanced Engineering Electromagnetics; John Wiley and Sons: Hoboken, NJ, USA, 1999.

13. Kong, J.A. Electromagnetic Wave Theory; EMW Publishing: Cambridge, UK, 2008.

(C) 2019 by the authors. Licensee MDPI, Basel, Switzerland. This article is an open access article distributed under the terms and conditions of the Creative Commons Attribution (CC BY) license (http:/ / creativecommons.org/licenses/by/4.0/). 